\title{
Current possibilities of liver volume estimation in diagnostic ultrasound (ex vivo study)
}

\begin{abstract}
Ultrasound evaluation of the liver volume can be useful for clinicians to aid in objective quantitative assessment of the liver size. For some organs (for example thyroid gland, testicles, ovaries) in diagnostic radiology the volume is the most objective criterion for estimation its size. Today CT and MRI imaging modalities use an objective techniques for volume calculation of the liver. MRI and CT-based liver volumetry are regarded as gold standard for evaluation of the liver volume. ${ }^{1-4}$ Nevertheless currently the ultrasonic linear measurements being used clinically as an indicator of overall liver size. Up to date in clinical sonography the ultrasound report contains only the linear measurements of the liver. ${ }^{5}$ Surely this fact significantly reduces the diagnostic value of the clinical estimation of the liver size.
\end{abstract}

Volume 5 Issue 5 - 2018

\author{
VA Izranov,' AV Ermakov, ${ }^{2}$ MV Martinovich, ${ }^{3}$ \\ NV Kazantseva, ${ }^{4}$ IA Stepanyan ${ }^{1,4}$ \\ 'Immanuel Kant Baltic Federal University, Kaliningrad, Russia \\ ${ }^{2}$ Bureau of Forensic Medical Examination of Kaliningrad Region, \\ Kaliningrad, Russia \\ ${ }^{3}$ Novosibirsk State Technical University, Novosibirsk, Russia \\ ${ }^{4}$ Infectious Diseases Hospital of Kaliningrad Region, Kaliningrad, \\ Russia
}

Correspondence: VA Izranov, Immanuel Kant Baltic Federal University, Kaliningrad, Russia, Email izranov@mail.ru

Received: July 30, 2018 | Published: September 27, 2018

\section{Introduction}

At present, the linear measurements of the liver are included in the actual diagnostic ultrasound scan protocol. The liver is commonly measured during a routine upper abdominal ultrasound. Measurement of liver size using ultrasound is most often determined by taking a simple linear measurements of the liver from a plane along the midclavicular line, and using cut off values to differentiate normal from abnormal liver size. ${ }^{6}$ One measurement of liver size is done in the mid-clavicular line from highest peak of the right hemidiaphragm down to the caudal liver end (maximal cranio-caudal diametr-Max $\mathrm{CC}$ ). This has a maximum dimension $18 \mathrm{~cm}$. Another possibility to measure the liver size is in the mid-clavicular line to measure craniocaudal dimension (CC) and antero-posterior dimension (AP). The maximum $\mathrm{CC}$ is $15 \mathrm{~cm}$ and $\mathrm{AP} 13 \mathrm{~cm}$, maximum for both dimensions together is $28 \mathrm{~cm} ., 7,8$

An attempts to estimate the liver volume on the basis of sonographic liver measurements have a long history. ${ }^{9-11}$ The complexity of calculating the liver volume is due to irregular geometric shape of the liver, which cannot be approximated to an ellipse. In systematic review of J. Childs et al. ${ }^{12}$ the methods of assessment of the liver size using 2D ultrasound are divided into 4 groups: volume measurements, body ratio measurements, representative measurements, and volumetric measurements. A volume measurement is a layer-bylayer measurement technique that resulted in an estimate of the overall volume of the liver. Body ratio measurements are those that measured the size of the liver as a ratio to body size. Representative measurements are those that do not reflect the true volume or size of the liver in more than one dimension. Volumetric measurements are those that use simple measurement techniques to develop a hepatic volumetric index (HVI) and then use a mathematical formula to translate this into a true liver volume. ${ }^{12}$

The calculations of the liver volume using an equations to convert simple sonographic measurements into a volume represent a group of volumetric methods. Volumetric techniques promise to be a quick and easy way to measure the liver.
In most volumetric methods the product of three mutually perpendicular dimensions of the organ are used. The calculating equation consist in product of simple liver measurements and the coefficients based on regression analysis. These methods were not widely used in clinical ultrasound due to the technical complexity to measure the accurate transverse dimension of the liver and the lack of generally accepted standards of the liver volume. In recent years, a new calculation formula of the liver volume was proposed..$^{13,14} \mathrm{New}$ formula does not require the transverse measurements of the liver. It is advantageous in that they are simple and rapid to perform, and can be reproduced easily on modern-day equipment. That is why it could be include into routine ultrasound examination.

The ability to convert simple liver measurements into a volume using an equation would be of great value in a clinical setting. Using multiple simple measurements to generate a liver volume would be superior to that of using only a single or several measurements, which gives a semiquantitative estimate of liver size. ${ }^{13}$

\section{The aim of the study}

Ex-vivo to assess the liver volume calculation volumetric formulae

\section{Tasks}

a. To estimate the liver weight after extraction from the cadaver abdominal cavity.

b. To estimate the liver volume using water displacement method.

c. To calculate the liver volume using clinical ultrasound volumetric formulae

d. To compare the results of the liver volume calculation with real liver volume and to select the optimal liver volumetric formula.

\section{Material and methods}

The study was performed on 34 cadavers of persons who died as a result of various diseases studied in the Bureau of forensic medical examination of the Kaliningrad region. All the forensic examination 
were performed in accordance with the Order of the health Ministry of the Russian Federation from may 12, 2010 \# 346-n "On approval of the procedure for the organization and production of forensic medical examinations at state forensic expert institutions of the Russian Federation". The corpses were studied by the method of Shor. Among the investigated corpses were 20 men and 14 women aged from 28 to 96 years. The cause of death are shown in Table 1.

Table I The cause of death and the number of observations ( $n$ )

\begin{tabular}{ll}
\hline The cause of death & $\mathrm{N}$ \\
\hline Pancreatic cancer & $\mathrm{I}$ \\
Obstructive asphyxia & $\mathrm{I}$ \\
Hemorrhagic stroke & $\mathrm{I}$ \\
Hanging & $\mathrm{I}$ \\
Burn disease at the stage of septicotoxemia & $\mathrm{I}$ \\
Pulmonary embolism & $\mathrm{I}$ \\
Hypothermia & $\mathrm{I}$ \\
Empyema & $\mathrm{I}$ \\
Urinary bladder cancer & $\mathrm{I}$ \\
Bilateral subtotal pleuropneumonia & $\mathrm{I}$ \\
Poisoning by unknown substance & $\mathrm{I}$ \\
Ischemic cardiomyopathy & $\mathrm{I}$ \\
Cirrhosis of the liver. & 2 \\
Acute myocardial infarction & 2 \\
Alcohol cardiomyopathya & 3 \\
Atherosclerotic heart disease & $\mathrm{I}$ \\
\hline
\end{tabular}

During the forensic autopsy the liver was extracted from the abdominal cavity. After the removal of the gallbladder and ligaments the liver were weighed and then placed in a container of water to determine body volume by measuring the volume of displaced fluid.

Measurement of the liver size was performed on the liver specimens according the rules to require liver diameters in clinical ultrasound. ${ }^{7}$ For this purpose we carried out two sections of the liver specimen in parasagittal plane. Right lobe was dissected at the level of the highest point of the diaphragmatic surface (Figure 1). The left lobe was dissected close to the falciform ligament (Figure 2).
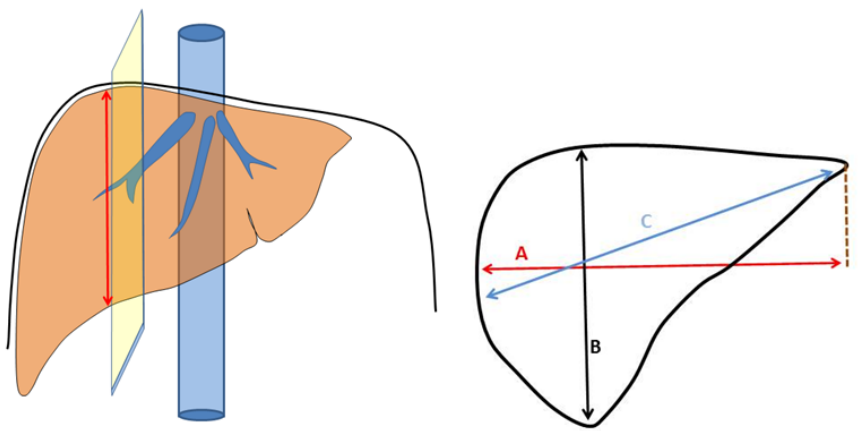

Figure I Schematic representation of the liver right lobe section to measure the cranio-caudal size of the right lobe (CCRL, $A)$, antero-posterior diameter of the right lobe (APRL, B), and maximal cranio-caudal diameter of the right lobe (maxCCRL).

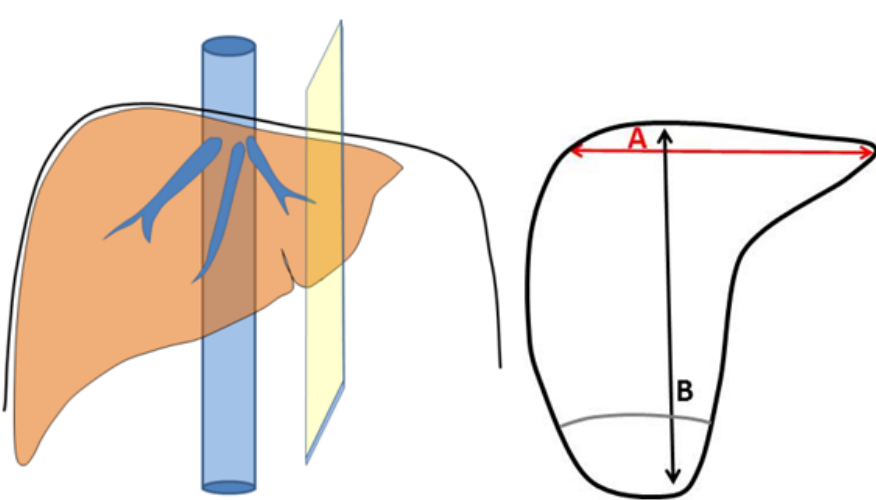

Figure 2 Schematic representation of the liver left lobe section the to measure the cranio-caudal size of the left lobe (CCLL,A) and antero-posterior diameter of the left lobe (APLL, B).

Liver width was measured at the outermost lateral points of the two lobes in horizontal plane (Figure 3). Microsoft Excel software was used for analyzing the data. For continuous variables, mean, maximal, minimal values and standard deviation was used. The volume difference between the calculated liver volume (CLV) on the base of each liver volumetric formula and actual liver volume (ALV) estimated using water displacement method was demonstrated with the percentage error [(CLV-ALV)/ALVx100].

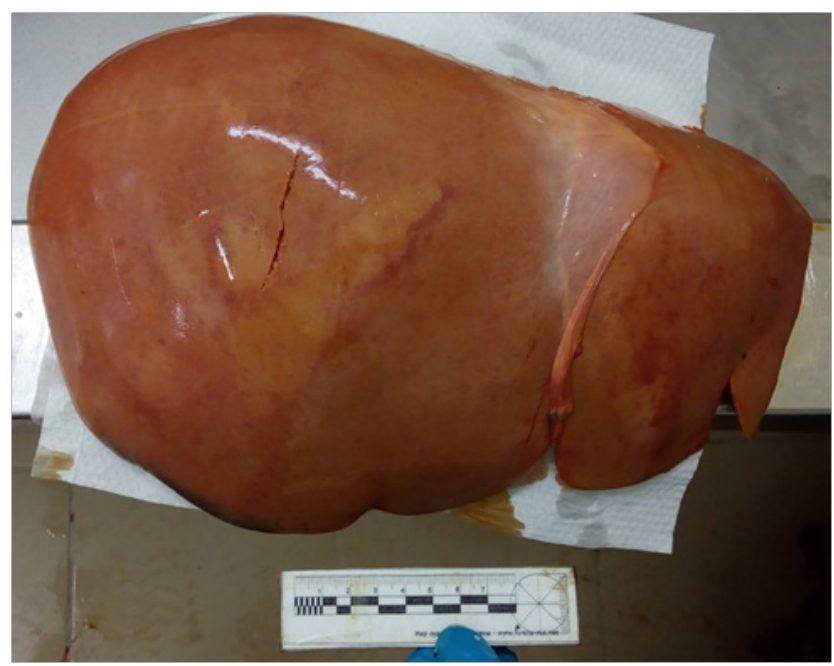

Figure 3 The width measurement of the liver.

\section{Results}

The average weight of liver according to results of post-mortem weighing was $1607 \pm 562 \mathrm{~g}$. The average volume of the liver estimated using water displacement method, was $1434 \pm 503 \mathrm{ml}$. In all cases, the liver weight (in grams) exceeds the amount of liver volume (in milliliters). The average density of liver tissue was $1.14 \pm 0.16 \mathrm{~g} \backslash \mathrm{ml}$.

Mean values of liver volume were calculated using five formulas. ${ }^{13,15-19}$

The average liver volume calculated using formula of JT Childs et al., ${ }^{14}$ was $1355 \pm 487$ (range 668-2631)ml. The results of the measurements and calculated liver volume are presented in Table 2. 
Table 2 The post-mortem weight of the liver and the LVC using different available formulae. The percentage of deviation of calculated liver volume in comparison with the actual volume of the liver estimated using water displacement method

\begin{tabular}{|c|c|c|c|c|c|c|c|c|c|c|c|c|}
\hline \multirow[b]{2}{*}{ 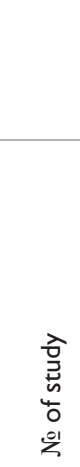 } & & & \multicolumn{5}{|c|}{$\begin{array}{l}\text { Liver volume calculated using various volumetric } \\
\text { formulae. }(\mathrm{ml})\end{array}$} & \multicolumn{5}{|c|}{$\begin{array}{l}\text { The percentage of deviation of calculated liver } \\
\text { volume in comparison with the actual volume } \\
\text { of the liver estimated using water displacement } \\
\text { method }\end{array}$} \\
\hline & 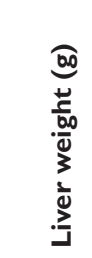 & 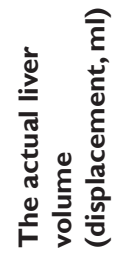 & $\begin{array}{l}\overline{0} \\
\check{c} \\
\frac{0}{\cup}\end{array}$ & $\sum_{\substack{n \\
N}}^{n}$ & 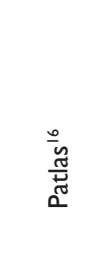 & 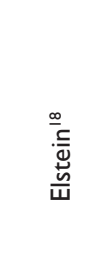 & $\frac{m}{\frac{m}{n}}$ & 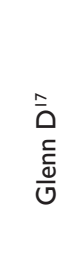 & $\sum_{\substack{0 \\
N}}^{n}$ & $\frac{0}{\frac{0}{d}}$ & $\begin{array}{l}\frac{\infty}{C} \\
\stackrel{C}{\mathbb{U}} \\
\frac{\omega}{W}\end{array}$ & $\frac{m}{\frac{m}{n}}$ \\
\hline I & 2121 & 1840 & 2640 & $294 I$ & 2867 & 2455 & 1547 & 43 & 60 & 56 & 33 & -16 \\
\hline 2 & 1015 & 620 & 1222 & 1448 & 1343 & 1309 & 990 & 97 & 134 & 117 & 111 & 60 \\
\hline 3 & 2593 & 2000 & 2303 & 2611 & 2531 & 2182 & 1183 & 15 & 31 & 27 & 9 & $-4 I$ \\
\hline 4 & 798 & 672 & 934 & 1104 & 991 & 1076 & 863 & 39 & 64 & 47 & 60 & 28 \\
\hline 5 & $|80|$ & 1697 & 1675 & 1369 & 1262 & 1675 & 1285 & -1 & -19 & -26 & -1 & -24 \\
\hline 6 & 1400 & 1180 & $|56|$ & 1306 & 1198 & 1583 & 1136 & 32 & II & 2 & 34 & -4 \\
\hline 7 & 1279 & 1100 & 2316 & 1596 & 1494 & 2193 & 1505 & 111 & 45 & 36 & 99 & 37 \\
\hline 8 & 1736 & 1650 & 2620 & 2129 & 2038 & 2438 & 1637 & 59 & 29 & 24 & 48 & -1 \\
\hline 9 & 1152 & 950 & 1620 & 1364 & 1257 & 1631 & 1225 & 71 & 44 & 32 & 72 & 29 \\
\hline 10 & 2143 & 1800 & 3209 & 2477 & 2393 & 2915 & 2018 & 78 & 38 & 33 & 62 & 12 \\
\hline II & 1669 & 1425 & 3129 & 2004 & 1910 & 2850 & 1700 & 120 & 41 & 34 & 100 & 19 \\
\hline 12 & 826 & 690 & 895 & 841 & 722 & 1044 & 687 & 30 & 22 & 5 & 51 & 0 \\
\hline 13 & 2300 & 2080 & 3017 & 2631 & 2551 & 2760 & 2136 & 45 & 26 & 23 & 33 & 3 \\
\hline 14 & 1134 & 980 & 1675 & 1170 & 1059 & 1674 & II 77 & 7I & 19 & 8 & 7I & 20 \\
\hline 15 & 2711 & 2475 & 4669 & 3288 & 3222 & 4095 & 2631 & 89 & 33 & 30 & 65 & 6 \\
\hline 16 & 1982 & 1935 & 2591 & 1894 & 1798 & 2415 & 1133 & 34 & -2 & -7 & 25 & $-4 I$ \\
\hline 17 & 2103 & 1980 & 3371 & 2217 & 2128 & 3046 & 1785 & 70 & 12 & 7 & 54 & -10 \\
\hline 18 & 1765 & 1460 & 2882 & 1712 & 1612 & $265 I$ & 1689 & 97 & 17 & 10 & 82 & 16 \\
\hline 19 & 1657 & 1577 & 1643 & 2106 & 2015 & 1649 & 1079 & 4 & 34 & 28 & 5 & -32 \\
\hline 20 & 1335 & 1210 & 1452 & 1085 & 972 & 1495 & 976 & 20 & -10 & -20 & 24 & -19 \\
\hline 21 & 909 & 695 & 1224 & 1107 & 994 & 1310 & 1063 & 76 & 59 & 43 & 88 & 53 \\
\hline 22 & 1725 & 1490 & 2352 & 1990 & 1896 & 2222 & $|52|$ & 58 & 34 & 27 & 49 & 2 \\
\hline 23 & $127 \mid$ & 1172 & 1669 & 1378 & $127 \mid$ & 1670 & 887 & 42 & 18 & 8 & 42 & -24 \\
\hline 24 & 1167 & 1011 & 1245 & 1032 & 918 & 1327 & 107| & 23 & 2 & -9 & 31 & 6 \\
\hline 25 & 1236 & 1070 & 2227 & 1484 & 1379 & 2121 & 977 & 108 & 39 & 29 & 98 & -9 \\
\hline 26 & 2047 & 1660 & $274 I$ & 2213 & 2124 & 2537 & 1666 & 65 & 33 & 28 & 53 & 0 \\
\hline 27 & 943 & 800 & 1097 & 1200 & 1089 & 1208 & 850 & 37 & 50 & 36 & 51 & 6 \\
\hline 28 & 2110 & 1923 & 3164 & 2897 & 2823 & 2878 & 2403 & 65 & 51 & 47 & 50 & 25 \\
\hline 29 & 913 & 824 & 1305 & 1076 & 962 & 1376 & 711 & 58 & 31 & 17 & 67 & -14 \\
\hline 30 & 744 & 1562 & 1229 & 1000 & 885 & 1315 & 668 & -21 & -36 & -43 & -16 & -57 \\
\hline 31 & 1562 & 1430 & 2879 & 2534 & 2452 & 2648 & 1312 & 101 & 77 & 71 & 85 & -8 \\
\hline 32 & 1779 & 1530 & 2013 & 1743 & 1644 & 1948 & 1026 & 32 & 14 & 7 & 27 & -33 \\
\hline
\end{tabular}


Table Continued...

\begin{tabular}{|c|c|c|c|c|c|c|c|c|c|c|c|c|}
\hline \multirow[b]{2}{*}{33} & \multirow[b]{2}{*}{2015} & \multirow[b]{2}{*}{1977} & \multicolumn{5}{|c|}{$\begin{array}{l}\text { Liver volume calculated using various volumetric } \\
\text { formulae. (ml) }\end{array}$} & \multicolumn{5}{|c|}{$\begin{array}{l}\text { The percentage of deviation of calculated liver } \\
\text { volume in comparison with the actual volume } \\
\text { of the liver estimated using water displacement } \\
\text { method }\end{array}$} \\
\hline & & & 3854 & 2702 & 2623 & 3436 & 1883 & 95 & 37 & 33 & 74 & -5 \\
\hline 34 & 2706 & 2300 & 4369 & 3286 & 3220 & 3853 & 1640 & 90 & 43 & 40 & 68 & -29 \\
\hline$M$ & 1607 & 1434 & 2259 & $|85|$ & 1754 & 2147 & 1355 & 56 & 32 & 23 & 53 & I \\
\hline $\mathrm{CO}$ & 562 & 503 & 979 & 709 & 725 & 791 & 487 & 35 & 31 & 30 & 31 & 27 \\
\hline Min & 744 & 620 & 895 & 841 & 722 & 1044 & 668 & -21 & -36 & -43 & -16 & -57 \\
\hline Max & III & 2475 & 4669 & 3288 & 3222 & 4095 & 2631 & 120 & 134 & 117 & III & 60 \\
\hline
\end{tabular}

( $M$, arithmetic mean; SD, standard deviation; Min, Max, minimum and maximum value).

We have determined the percentage deviation of liver volume calculated based on volumetric formulae with respect to the actual volume of the liver estimated using water displacement method. It was discovered that the average percentage deviation of the calculated liver volume varies widely up to $56 \%$ formula ${ }^{17}$ compared to the AVL. The lowest percentage average deviation (1\%) were found in the formula Childs JT. ${ }^{14}$ At the same time, the standard deviation of errors of calculation of the liver volume according to the formula is approximately the same (27\%) JT Childs,${ }^{14}$ up to $35 \%$ Glenn D. ${ }^{17}$

\section{Discussion}

\section{The liver volume and the liver weight}

In our study the liver weight (in grams) exceeds the amount of liver volume (in milliliters). Post-mortem extracted from the cadaveric corp liver specimen sinks when water displacement method occures. In our study it is shown that the weight and volume of the liver are not identical variables. The average value of the density of liver tissue determined as $1.14 \mathrm{~g} / \mathrm{ml}$, a standard deviation $-0.16 \mathrm{~g} / \mathrm{ml}$.

The issue of the density of the liver tissue has already been discussed the scientific literature. It was initiated by the fact that preoperative determination of the liver mass volume using CT-volumetry exceeds the actual volume of the resected mass, measured using water displacement method. ${ }^{20} \mathrm{SM}$ Niehues et al. ${ }^{21}$ carried out a pilot study aimed at a systematically determine the difference between the results of in vivo CT liver volumetry and ex vivo water displacement liver volumetry in a pig animal model. The authors showed that the median density of liver tissue is $1.07 \mathrm{~g} / \mathrm{ml}$. Regression analysis showed a high correlation $(\mathrm{r} 2=0,985)$ between the results of CT volumetry and the water displacement method. CT volumetry was found to be $13 \%$ higher than the water displacement volumetry $(\mathrm{p}<0.0001)$. The reason of difference between in vivo CT-volumetry and ex vivo water displacement volumetry seems to be blood perfusion of the liver.

K. Kitajima et al. ${ }^{22}$ analyzed the volume of the liver specimen calculated by the water-displacement method and the volume liver fragment determined preoperatively by ultrasonography. As the volume of each compartment assessed by ultrasonography included blood volume and there was no blood in the large vessels of the resected specimens they assumed that the ultrasonography-visible vascular bed represented $15 \%$ of the whole liver volume and, thus, added $15 \%$ increment to the specimen volume before comparison. The percentage difference between the ultrasonography volume and specimen vo;ume (plus 15\% increment) was calculated according the equation: (ultrasonography volume - specimen volume*1,15)/ specimen volume $* 100)$.

It is known that in the norm $100 \mathrm{~g}$ of liver tissue contains 25$30 \mathrm{ml}$ of blood. ${ }^{23,24}$ However, the exact distribution of blood volume between the large and small intrahepatic vessels is still not known, as it is not known what amount of blood is visualized in the intrahepatic vessels at sonography, and accordingly, the extent to which this affects the volume of the organ. Normally, $100 \mathrm{~g}$ of liver contains $25-30 \mathrm{ml}$ of blood. ${ }^{23,24}$ However the respective distribution of blood volume between the intrahepatic large and small vessels is not clear, no is amount of blood in the intrahepatic vascular bed, vizulised by echography. ${ }^{22}$

In our study, the mean density of liver tissue was $1.14 \pm 0.16 \mathrm{~g} / \mathrm{ml}$, which exceeds the same results of Niehues SM et al. ${ }^{21}$ Surely, the liver of pigs and humans may have different density of the tissue. However, O Garkavenko et al. ${ }^{25}$ it was shown that healthy liver tissue has a density in the range of 1.02 to $1.09 \mathrm{~g} / \mathrm{ml}$, which corresponds to the results of Niehues SM. ${ }^{21}$ We assume that higher average values of the liver tissue density in our study is due to the significant number of the studied specimen were affected diffuse disease or focal lesions of the liver, that also affects the density of the liver tissue.

\section{Comparison of the formulas}

Table 3 lists used in this article formulae to calculate the volume of the liver. When analising the dates of publications we can conclude that the problem of determining liver volume was actual in 1989 and remains relevant nowdays. Why in the daily practice of ultrasonographers these formulas do not apply, despite the high relevance of the liver size assessment? One of the answers to this issue can be found in the line "M" of table No. 3 (mean error of the formula). The average percent deviation from ALV varies from $23 \%{ }^{16}$ to $56 \% .{ }^{17}$ This is an unsatisfactory high values of the average percent error formula. The exception is the formula J Childs, ${ }^{14}$ which shows the minimum average deviation from actual volume (1\%). However, the authors in their article ${ }^{13}$ point to the insufficient accuracy of the formula. This is caused in particular by the standard deviation of the average error. All the formulae in our study demonstrate similar hight variation up to $35 \%$ as well as the formula of J. Childs - $27 \%$ (Table 3 ). We should also note that all the formulae can give quite a large error in a big way. This is unacceptable from a diagnostic point of view. According to this parameter formula of Childs $\mathrm{JT}^{14}{ }^{14}$ is significantly, 1.66-2.23 times better than the other formulae. 
Table 3 Shows the most common formulas of the liver volume calculation based on the clinical ultrasound measurements

$\begin{array}{ll}V=133,2+0,422 \times C C R L \times A P R L \times T r a n s L & \text { M. Zoli }(1989) \\ V=(C C R L \times A P R L \times T r a n s L-545) / 2320 & \text { M.Patlas e.a. }(200 I) \\ V=(0,12+C C R L \times A P R L \times T r a n s L) / 2,55 & \text { D. Glenn e.a. }(1997) \\ V=320,86+0,3 I 7 \times C C R L \times A P R L \times T r a n s L & \text { D. Elstein e.a. }(1997) \\ V=345,7 I+0,84 \times \operatorname{maxCCRL} \times A P R L \times A P L L & \text { J.Childs e.a. }(2014,2016)\end{array}$

(CCRL, cranio-caudal diameter of the right lobe; APRL, antero-posterior diameter of the right lobe; TransL, transversal diameter of the liver; maxCCRL, maximal cranio-caudal diameter of the right lobe; APLL, antero-posterior diameter of the left lobe.All dimensions are in centimeters).

The second answer to the question why CLV formulae are not used in clinical ultrasound, is due to technical difficulties and operatordependence of the measurement process. Four of five CLV formulae listed in table 1 use width of the liver as one of the arguments of the equation. Fig. 3 demonstrates the method of measurement of the width of the liver on the specimen. To measure TransL through clinical ultrasound using convex probe in most cases is impossible (Figure 4). Even when liver has a normal size its lateral borders are outside of the working area of the monitor screen. From this point of vue, the bias of TransL measurement in clivical ultrasound is unsatisfactory high.

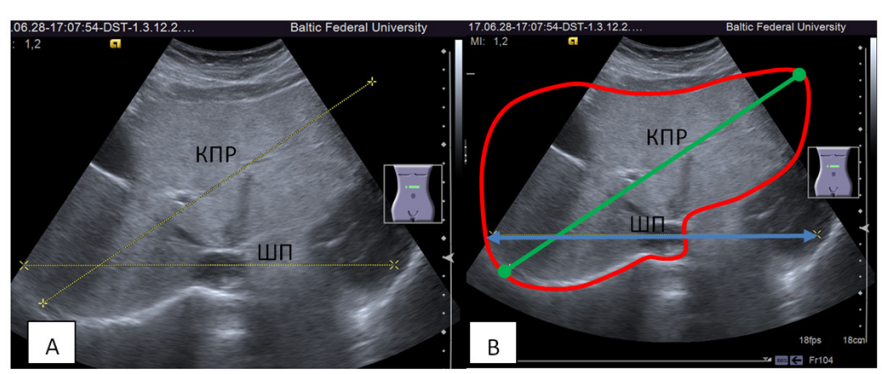

Figure $4 \mathrm{~A}$ : Ultrasound cross section of the liver trough subcostal access. ШП, width of the liver measured in horizontal plane (TransL), КПР, oblique transverse diameter (the most remote point of the right dome of the diaphragm and the left border of the liver). B:The same image. The borders of the liver are outlined.The ШП (TransL) do not fit in the image.

After publication of JT Childs, ${ }^{13,14}$ new volumetric formula has appeared. Liver volumetry now does not require additional time and special training of sonographer, because new volumetric equation consist in three simple liver diameters and does not use TransL. These liver measurements are recommended in last training course for clinical ultrasound and are included in ultrasound protocol (EFSUMB).

The next reason why volumetric formulae for calculation liver volume are not widely used in clinical ultrasound, is the lack everywhere spread of automated logging of the results of the ultrasound. Surely, the calculation of the liver volume on the calculator is an unacceptable complication of a routine process of logging of the clinical ultrasound results. The liver volumetric formulae should be built in automated workstation and in ultrasound equipment.

Figure 5 demonstrates a screenshot of the automated module for the liver volume assessment we have proposed to include in automated workstation for clinical ultrasound "Assistant." We believe that the built-up of liver volumetric formulae in ultrasound equipment is an important factor for the objectiveness of the liver size estimation in clinical ultrasound.

\section{Estimation of the liver volume}

In present study we did not carry out to estimate the "normality" of the liver volume. We suppose the optimal formula must be able to accurately calculate the volume of the normal liver, and also of enlarged or reduced liver. Determination of the standard ranges of the liver volume, including constitutional features of the patients, is a topic of further research.

It is interesting to compare the actual liver volume with the standard liver volume. Unfortunately, in our study for technical reasons it was not possible to weigh the cadaver. In this regard, it is impossible to calculate the standard liver volume due to the lack of data on body weight. Earlier in our article, ${ }^{26}$ a comparative analysis of the accuracy of 14 published formulae of the standard liver volume calculation was carried out. ${ }^{16,27,28}$ There is a perception that on the amount of the standard liver volume racial-ethnic and territorialgeographical factors are influenced. This is because the formulae for calculating standard liver volume are based on anthropometric data, which have ethno-territorial dependence. In work, ${ }^{26}$ it was shown that the most accurate calculation formula of standard liver volume for use in conditions of the Kaliningrad region of the Russian Federation is the formula Chouker A. ${ }^{27}$ This formula can be recommended as a reference evaluation for estimation CLV according to J. T. Childs. As the results of present study, we propose to include the formulae of JT Childs ${ }^{16}$ \& A Chouker ${ }^{27}$ into the clinical ultrasound protocol to calculate the volume of the liver and its reference evaluation.

\section{Conclusion}

a. The average weight of liver as the results of post-mortem weighing is $1607 \pm 562 \mathrm{~g}$.

b. The average liver volume determined by the water displacement method, is $1434 \pm 503 \mathrm{ml}$.

c. The average density of liver tissue is $1.14 \pm 0.16 \mathrm{~g} / \mathrm{ml}$.

d. The average calculated liver volume according to the formulae Glenn D, ${ }^{17}$ Zoli M, ${ }^{15}$ Patlas M, ${ }^{16}$ Elstein D. ${ }^{18}$ and Childs JT, ${ }^{16}$ amounted to $2259 \pm 979,1851 \pm 709,1754 \pm 725,2147 \pm 791$ and $1355 \pm 487 \mathrm{ml}$, respectively.

e. The optimal formula to calculate liver volume in terms of the least deviation from the actual liver volume is Childs $\mathrm{JT}^{12,13}$ formula.

\section{Acknowledgements}

None.

\section{Conflict of interest}

The author declares that there is no conflict of interest.

\section{References}

1. Borchert D, Schuler A, Muche R, et al. Comparison of Panorama Ultrasonography, Conventional B-Mode Ultrasonography, and Computed Tomography for Measuring Liver Size. Ultrashall in Med. 2010;31(1):3136.

2. Bora A, Alptekin C, Yavuz A, et al. Assessment of liver volume with computed tomography and comparison of findings with ultrasonography. Abdom Imaging. 2014;39(6):1153-1161.

3. D’Onofrio M, De Robertis R, Demozzi E, et al. Liver volumetry: Is imaging reliable? Personal experience and review of the literature. WJR. 2014;6(4):62-71. 
4. Xiaoqi Lv, Yu Miao, Xiaoying Ren, et al. The study and implementation of liver volume measuring method based on 3-dimensional reconstruction technology. Optik. 2015;126(17):1534-1539.

5. Roger C Sanders, Thomas C Winter III, Teresa Bieker, et al. Clinical sonography: a practical guide. 4th ed. USA: Lippincott Williams \&Wilkins; 2007:724

6. Childs JT, Esterman AJ, Thoirs KA, et al. Ultrasound in the assessment of hepatomegaly: A simple technique to determine an enlarged liver using reliable and valid measurements. Sonography. 2016;3(2):47-52.

7. Dietrich CF, Tuma J, Badea R. Ultrasound of the liver EFSUMB. European Course Book. 2013:65.

8. Abraham D, Silkowski C, Odwin C. Emergency Medicine Sonography: Pocket Guide to Sonographic Anatomy and Pathology. Jones and Bartlett Publishers. 2009:308.

9. Rasmussen SN. Liver volume determination by ultrasonic scanning. $B r J$ Radiol. 1972;45(536):579-585.

10. Carr D, Duncan JG, Railton R, et al. Liver volume determination by ultrasound: a feasibility study. Br J Radiol. 1976;49(585):376-778.

11. Raeth U, Johnson PJ, Williams R. Ultrasound determination of liver size and assessment of patients with malignant liver disease. Liver. 1984;4(5):287-293

12. Childs JT, Esterman A, Thoirs KA. Ultrasound measurements of the liver: an intra and inter-rater reliability study. Australian journal of ultrasound in medicine. 2014;17(3):113-119.

13. Childs JT, Esterman AJ, Thoirs KA. The development of a practica and uncomplicated predictive equation to determine liver volume from simple linear ultrasound measurements of the liver. Radiography. 2016;22(2):125-130

14. Childs JT, Esterman AJ, Phillips M, et al. Methods of determining the size of the adult liver using 2D ultrasound: a systematic review of articles reporting liver measurement techniques. J Diagn Med Sonogr. 2014;30(6):296-306

15. Zoli M, Pisi P, Marchesini G, et al. A rapid method for the in vivo measurement of liver volume. Liver. 1989;9(3):159-163.

16. Patlas M, Hadas-Halpern I, Abrahamov A, et al. Spectrum of abdominal sonographic findings in 103 pediatric patients with Gaucher disease. Eur Radiol. 2002;12(2):397-400.
17. Glenn D, Thurston D, Garver P, et al. Comparison of magnetic resonance imaging and ultrasound in evaluating liver size in Gaucher patients. Acta Haematol. 1994;92(4):187-189.

18. Elstein D, Hadas-Halpern I, Azuri U, et al. Accuracy of ultrasonography in assessing spleen and liver size in patients with Gaucher disease: comparison to computed tomographic measurements. J Ultrasound Med. 1997;16(3):209-211.

19. Frericks BB, Caldarone FC, Nashan B,. et al. 3D CT modeling of hepatic vessel architecture and volume calculation in living donated liver transplantation. Eur Radiol. 2004;14:326-333.

20. Hiroshige S, Shimada M, Harada N, et al. Accurate preoperative estimation of liver-graft volumetry using three-dimensional computed tomography. Transplantation. 2003;75:1561-1564.

21. Niehues SM, Unger JK, Malinowski M, et al. Liver volume measurement: reason of the difference between in vivo CT-volumetry and intraoperative ex vivo determination and how to cope it. Eur J Med Res. 2010;15:345350 .

22. Kitajima K, Taboury J, Boleslawski E, et al. Sonographic preoperative assessment of liver volume before major liver resection. Gastroenterol Clin Biol. 2008;32(4):382-389.

23. Lautt WW. Hepatic vasculature: a conceptual review. Gastroenterology. 1977;73(5):1163-1169.

24. Hwang S, Lee SG, Kim KH, et al. Correlation of blood-free graft weight and volumetric graft volume by an analysis of blood content in living donor liver grafts. Transplant Proc. 2002;34:3293-3294.

25. Garkavenko O, Emerich D, Muzina M, et al. Xenotransplantation of neonatal porcine liver cells. Transplant proc. 2005;37(1):477-480.

26. Izranov VA, Kazantseva NV, Beletskaya MA, et al. Estimating the Accuracy of Standard Volume Calculations Using Ultrasound Liver Volumetry. Scientific Journal. 2017:37-49.

27. Chouker A, Martignoni A, Martin Dugas M, et al. Estimation of Liver Size for Liver Transplantation: The Impact of Age and Gender. Liver Transpl. 2004;10(5);678-685.

28. Leung NW, Farrant P, Peters TJ. Liver volume measurement by ultrasonography in normal subjects and alcoholic patients. $J$ Hepatol. 1986;2(2):157-164 